\title{
Spinal and Supraspinal Contributions to Central Sensitization in Peripheral Neuropathy
}

\author{
Rie Suzuki Anthony Dickenson \\ Department of Pharmacology, University College London, London, UK
}

\author{
Key Words \\ $5 \mathrm{HT} \cdot 5 \mathrm{HT} 3 \cdot$ Spinal cord · Lamina I - Gabapentin • \\ Descending excitation $\cdot$ NK1 receptor $\cdot$ Substance $P$
}

\begin{abstract}
We will focus on spinal cord dorsal horn lamina I projection neurones, their supraspinal targets and involvement in pain processing. These spinal cord neurons respond to tonic peripheral inputs by wind-up and other intrinsic mechanisms that cause central hyper-excitability, which in turn can further enhance afferent inputs. We describe here another hierarchy of excitation - as inputs arrive in lamina I, neurones rapidly inform the parabrachial area (PBA) and periaqueductal grey (PAG), areas associated with the affective and autonomic responses to pain. In addition, PBA can connect to areas of the brainstem that send descending projections down to the spinal cord establishing a loop. The serotonin receptor, $5 \mathrm{HT} 3$, in the spinal cord mediates excitatory descending inputs from the brainstem. These descending excitatory inputs are needed for the full coding of polymodal peripheral inputs from spinal neurons and are enhanced after nerve injury. Furthermore, activity in this serotonergic system can determine the actions of gabapentin (GBP) that is widely used in the treatment of neuropathic pain. Thus, a hierarchy of separate, but interacting excitatory systems exist at peripheral, spinal and supraspinal sites that all con-
\end{abstract}

verge on spinal neurones. The reciprocal relations between pain, fear, anxiety and autonomic responses are likely to be subserved by these spinal-brainstem-spinal pathways we describe here. Understanding these pain pathways is a first step toward elucidating the complex links between pain and emotions.

Copyright (C) 2005 S. Karger AG, Basel

\section{Introduction}

Pain research is an advancing field of neuroscience asking questions about pain perception and basic neurobiological transmission in terms of how the central nervous system (CNS) processes sensory information. In terms of medical and social issues, a yet more important aspect of this research is knowledge that could improve the treatment of pain, particularly chronic pain. A fundamental approach to understand pain processing is to define the neuronal networks that mediate and modulate sensory inputs, and how trauma and disease may alter their function. Noxious stimulation evokes changes in many systems within the CNS; different chronic pain states generate unique pharmacological patterns in the brain. Models of pain in integrated systems are essential to this goal and our main hypothesis is that different pain states evoke different changes. New insights into how sensory information is centrally processed are fundamental

\section{KARGER}

Fax +41613061234 E-Mail karger@karger.ch www.karger.com
(C) 2005 S. Karger AG, Basel

1424-862X/05/0144-0175\$22.00/0

Accessible online at:

www.karger.com/nsg
Rie Suzuki, PhD

Department of Pharmacology, Medical Sciences Building

University College London, Gower Street

London WC1E 6BT (UK)

Tel. +44 207679 3737, Fax +44 207679 3742, E-Mail ucklrsu@ucl.ac.uk 
to better pain control and development of new analgesics.

As pain persists there are the affective and emotional responses that must be considered along with the sensory aspects of the stimulus. It is clear that although the sensory and psychological aspects of pain are separable, the neural pathways that contribute to these aspects of pain are inter-linked. Furthermore, at both peripheral and central sites, there are mechanisms that can amplify and prolong the painful stimulus so that the pain becomes greater - this can result in severe pain in the presence of relatively minor peripheral pathology. There is considerable capacity for change in both the transmission and modulating systems in these prolonged pain states, and plasticity occurs at both peripheral and central sites. Recently, a lot of attention has been given to the expression of genes in chronic pain models [1] - this may well pay dividends in terms of novel targets; however, it is equally likely that enhanced activity of constitutive systems could activate novel signalling pathways to alter pain levels markedly without the production of new proteins. This review will discuss changes in the central processing of noxious inputs that we believe to have marked and selective influences on both the level of pain signalling and the actions of therapeutic agents.

Pain starts in the periphery; acute pain causes the activation of peripheral C-fibres by activating nociceptors that respond to noxious mechanical, chemical or thermal stimuli. Peripheral events that accompany nerve injury are quite different from peripheral sensitization seen in inflammation, and it is paradoxical that damage to peripheral nerves not only give rise to expected negative symptoms (sensory deficits) but a proportion of patients report positive phenomena (abnormal spontaneous or evoked sensations). There is substantial evidence to suggest that alterations in somatosensory processing following nerve injury originate in the peripheral nervous system and these have been reviewed extensively [2]. Neuropathic pain, however, can be explained only partly by peripheral changes because patients can have severe pain yet also marked sensory loss - thus compensations within the central nervous system appear to be of major importance [3].

\section{Central Spinal Excitatory Events}

The first synapse in the transmission of noxious information from the periphery to the brain is in the superficial dorsal horn of the spinal cord, which is comprised of lam- ina I and II (the marginal zone and substantia gelatinosa, respectively) [4]. Lamina I of the spinal cord plays a key role in the modulation of pain transmission and these neurons have distinct response properties compared to deeper lying neurones (lamina V). Electrophysiological recordings of lamina I neurons show that the majority of these neurons (around 75\%) are nociceptive-specific (NS) in their responses, about $15 \%$ are termed polymodal nociceptive (HPC) with an additional response to noxious cold, and the remainder (10\%) are wide dynamic range (WDR). In lamina V the large majority are WDR so that information transmitted from these two major groups of dorsal horn neurons are almost entirely nociceptive in lamina I while lamina V neurons code in the innocuous through the noxious range. Lamina I neurons exhibit higher thresholds for excitation and generally have smaller mechanical and heat-evoked responses and receptive fields when compared to deeper dorsal horn neurons.

Superficial dorsal horn neurons are morphologically diverse. Lamina I neurons typically have long axons thus allowing projection to higher CNS centers [5]. This is not the case for lamina II, which is comprised mainly of small intrinsic neurons terminating locally [6]. A small proportion of lamina I neurons appears to project contralaterally via the lateral spinal thalamic tract (STT), which is a key pathway for pain, itch and temperature [7, 8], however the predominant ascending output from lamina I neurons appears to be the spino-parabrachial pathway in the rat $[5,9,10]$. Neurons in the deep dorsal horn (lamina V-VI), however, have predominant projections in the STT. The spinothalamic pathway is important for the sensory discriminatory aspects of nociception, whilst the spino-parabrachial pathway plays a key role in affective reactions to pain mechanisms and in regulating descending control systems [11].

Neurons in the parabrachial area (PBA) show specific activation by noxious stimuli and generally have large receptive fields covering a number of body parts [12]. This lack of somatotopy suggests roles in diffuse responses to pain and damage, rather than fine and localized information [13]. The two main forebrain targets of parabrachial neurons are the nucleus centralis of the amygdala and the ventrolateral medial hypothalamus [14]. Projections to the amygdala probably contribute to some components of aversive emotions such as fear and anxiety, whilst the PBA-hypothalamus-PAG circuits may be involved in aversive behaviors such as rage and aggression [12]. The PAG is closely associated with areas of the brainstem including the rostral ventromedial medulla ( RVM), and is critical in the descending modulation of 
spinal activity through monoaminergic and other pathways $[15,16]$. The PAG and RVM can exert both facilitatory and inhibitory influences on the spinal cord $[17,18]$. That these influences can be modulated by different manipulations suggests they involve anatomically distinct pathways [19]. The net balance of these two supraspinal pathways, together with primary afferent input, will ultimately determine the excitability of spinal neurons. Thus, the PBA receives specific noxious inputs from numerous spinal neurons then relays to brainstem sites that reciprocally project back to the spinal cord. These interacting pathways will form complex loops so the brain is first informed of spinal nociceptive activity but further regulation of spinal responses could be produced by the descending pathways [16].

The arrival of sensory information from nociceptors into the dorsal horn considerably alters the level of activity within the cord since both excitatory and inhibitory systems can impinge upon spinal neuronal activity. These influences can originate both intrinsically and supraspinally. Calcium channels on afferent terminals and neurons are important for both transmitter release and determining neuronal excitability. Augmented release of afferent transmitters, including peptides such as calcitonin gene-related peptide (CGRP) or substance P (SP), together with glutamate allows changes in their postsynaptic receptor actions. One of the key events is the activation of the N-methyl- $D$-aspartate (NMDA) receptor for glutamate that underlies wind-up, whereby the baseline response to a constant noxious input is amplified and prolonged [20,21]. This forms the basis of central hypersensitivity which results in an increased responsiveness of dorsal horn neurons, often observed in persistent inflammatory and neuropathic pain states. Recent data point to yet another type of facilitatory drive from within the brainstem (in particular, the RVM) that can enhance spinal mechanisms of pain, independently in some cases or alongside those mentioned previously. There is accumulating evidence for the contribution of these brainstem excitatory influences in the development and maintenance of central sensitization in persistent pain states (for review, see [17]). Here we emphasize systems that arise from lamina I neurons of the spinal dorsal horn that link to descending pathways from the brainstem. We will consider the relation between sensory inputs and emotional areas of the brain and the mechanisms by which central pharmacological systems can enhance pain.

\section{Lamina I Pathways and Descending Facilitation}

We have recently shown that selective ablation of lamina I NK1 receptor expressing neurons through the use of a SP-cytotoxin conjugate (SP-SAP) [22] produces profound effects on the response characteristics of deep dorsal horn neurons [23]. Approximately $45 \%$ of neurons in lamina I appear to express NK1 receptors [24] and ablation of these neurons resulted in (a) a reduction in receptive field size; (b) disruption in mechanical and thermal coding; (c) reduced responses to chemical inflammation, and (d) reduced central sensitization of deep dorsal horn neurons as revealed by diminished wind-up. These lamina I neurons therefore link peripheral afferent stimuli to the sensory coding of deep WDR neurons, and provide a major input to the brain [23]. As these cells form part of an important ascending pathway to the brainstem, one reason for the reduced responsiveness observed in deep dorsal horn neurons following SP-SAP treatment is likely to be due to the loss of the lamina I-PBA pathway. The latter may form part of a loop back to the spinal cord, and appears to be supported by pharmacological evidence. The anatomical and pharmacological evidence for descending pain facilitatory pathways is mounting; activation of the excitatory 5HT3 receptors in the spinal cord by serotonin released from pathways projecting from the RVM has been suggested to underlie the descending excitatory drive from the RVM to the spinal cord [23]. The $5 \mathrm{HT} 3$ receptors are predominantly localized in the superficial dorsal horn $[25,26]$ on nerve terminals of small diameter afferents [27]. Blocking spinal 5HT3 receptors using the selective antagonist ondansetron (Zofran ${ }^{\mathrm{TM}}$ ) and related drugs has implicated a pronociceptive role of these receptors [28, 29]. Importantly, most of the effects of ablating these lamina I neurones were reproduced by blocking descending serotonergic facilitatory pathways using spinally administered ondansetron in unlesioned animals. Thus, NK1-lamina I projection neurons forms the origin of a spino-bulbo-spinal loop, and consequently control spinal excitability at least in part through the activation of a descending serotonergic pathway.

It has to be pointed out that analgesic effects of activation of this receptor have also been reported by some studies. The apparent discrepancies may be due to variable test doses of drugs, assays, species differences, or the possibility that the excitatory action of 5HT3 may induce diffuse noxious inhibitory controls (DNIC); excessive excitation could also trigger depolarizing block of neuronal activity. Recent data from 5HT3 knock-out mouse sup- 
port the pronociceptive role of this receptor in the spinal cord [23]. Only wind-up did not depend on this 5HT3 receptor-mediated pathway, thus confirming the importance of spinal circuits for this neuronal measure [23]. In fact, it is clear from the variety of preparations that have been used to study wind-up that this short term increase in excitability is an intrinsic spinal event seen in spinalized animals, slices and neuronal co-cultures [21]. This leads to the intriguing possibility that quite different messages may be sent to the emotional/autonomic areas of the brain compared to the sensory-discriminative zones. Neurons in lamina I of the spinal cord have projections to PBA/PAG whereas many deep cells project in the STT tract. NMDA-dependent wind-up is clearly seen in the large majority of deep dorsal horn cells yet is almost absent in lamina I cells. This indicates that spinothalamic inputs will be potentiated through spinal wind-up-like mechanisms, whereas afferent messages to PBA will not. This may consequently lead to dissociation between the emotional and sensory-discriminative aspects of pain. However, both neuronal types support LTP when highfrequency stimuli are given [30-32].

\section{Central Pain Pathways and Neuropathic Pain}

As already discussed, neuropathic pain is driven by multiple peripheral and spinal mechanisms [33-35]. In addition to these alterations, nerve injury leads to enhanced descending excitatory drive from the RVM to maintain the chronic pain states [36]. Disruption of ascending or descending pathways effectively blocks abnormal pain after neuropathy, supporting the idea that these descending facilitatory systems contribute to the development of pain that follows peripheral nerve injury [37]. Cells of the RVM fall into three types, one of which has been associated with pronociception, and these neurones have been proposed to be the source of descending facilitation from the RVM [38]. Hence one possibility for the abnormal pain associated with neuropathic pain is an inappropriate activation of these brainstem cells which would promote excitatory influences onto the spinal cord and facilitate nociception [37]. This may represent a central compensatory mechanism for the loss of normal sensory input that results as a consequence of peripheral nerve damage [34]. Under pathological conditions descending facilitatory controls to the spinal cord are likely to be enhanced and excitatory influences predominate to maintain spinal central sensitization. Given that the brainstem areas involved in these sensory controls are also implicated in emotions, sleep and autonomic responses, the well established links between these states and pain may well involve these same pathways and provide a basis for alterations in pain as a result of affective changes [39].

Both chemical and electrical stimulation could evoke facilitations at doses and currents less than those required to elicit inhibitions (see refs in [17]). Thus, the balance between serotonergic and non-serotonergic excitatory and inhibitory controls may be critical for determining the level of spinal activity.

\section{HT in Central Pain Pathways}

Currently, the main focus of 5HT3 receptor antagonists such as ondansetron has been on the clinical treatment of postoperative and chemotherapy-induced emesis, and only limited evidence exists for the use of this class of drugs in pain management [40]. Our recent findings of an increased efficacy of ondansetron on mechanical punctuate evoked responses following peripheral nerve injury favors the potential clinical use of this agent for the treatment of neuropathy, particularly in patients with tactile allodynia. A preliminary clinical study suggests that block of 5HT3 receptors does indeed have clinical utility in the treatment of pain (fig. 1) [59].

More recently, we observed that ondansetron exerts greater effectiveness after nerve injury compared to sham controls, particularly on mechanical punctate responses [60]. This was clearly shown by the robust inhibition of these responses following the administration of a low dose of ondansetron, which under normal conditions, produces little or no effect in control animals. Our results support the idea that supraspinal serotonergic inputs to the spinal cord may have sustained facilitatory influences to the spinal cord following injury to peripheral nerves. Although there is a downregulation in the 5HT3 receptor gene in injured L5 and L6 DRG in the spinal nerve ligation (SNL) injury model, the gene is not altered in the neighboring uninjured L4 [1] suggesting that this receptor could function to alter evoked pain in this model. An interesting finding was the observation that ondansetron produces more selective and robust actions on mechanical punctate inputs compared to thermal inputs, thus leaving the heat responses relatively intact. The 5HT3 receptor has been localized to a novel group of small diameter afferents that are not IB4 positive, non-peptidergic, contain very few vanilloid receptors (TRPV1), and a larger number of presumed A-delta afferent fibers [27]. In vitro, 16\% of A- 
Fig. 1. Peripheral nerve injury induces changes in both the peripheral and central nervous system. Spontaneous activity is generated at the site of injury and the DRG. Plasticity is seen in the expression and function of ion channels (e.g. $\mathrm{Na}^{+}$channels) and neurotransmitters (e.g. SP). Sprouting of sympathetic nerve fibers in the DRG act to sensitize peripheral afferents. increased $\mathrm{Ca}^{2+}$ channel function will result in enhanced neurotransmission thereby promoting sensory transmission in the spinal cord. Centrally, there is increased function of the NMDA receptor and enhanced activity in serotonergic pathways arising from the brainstem. All these mechanisms can contribute to the development of abnormal pain accompanying nerve injury.

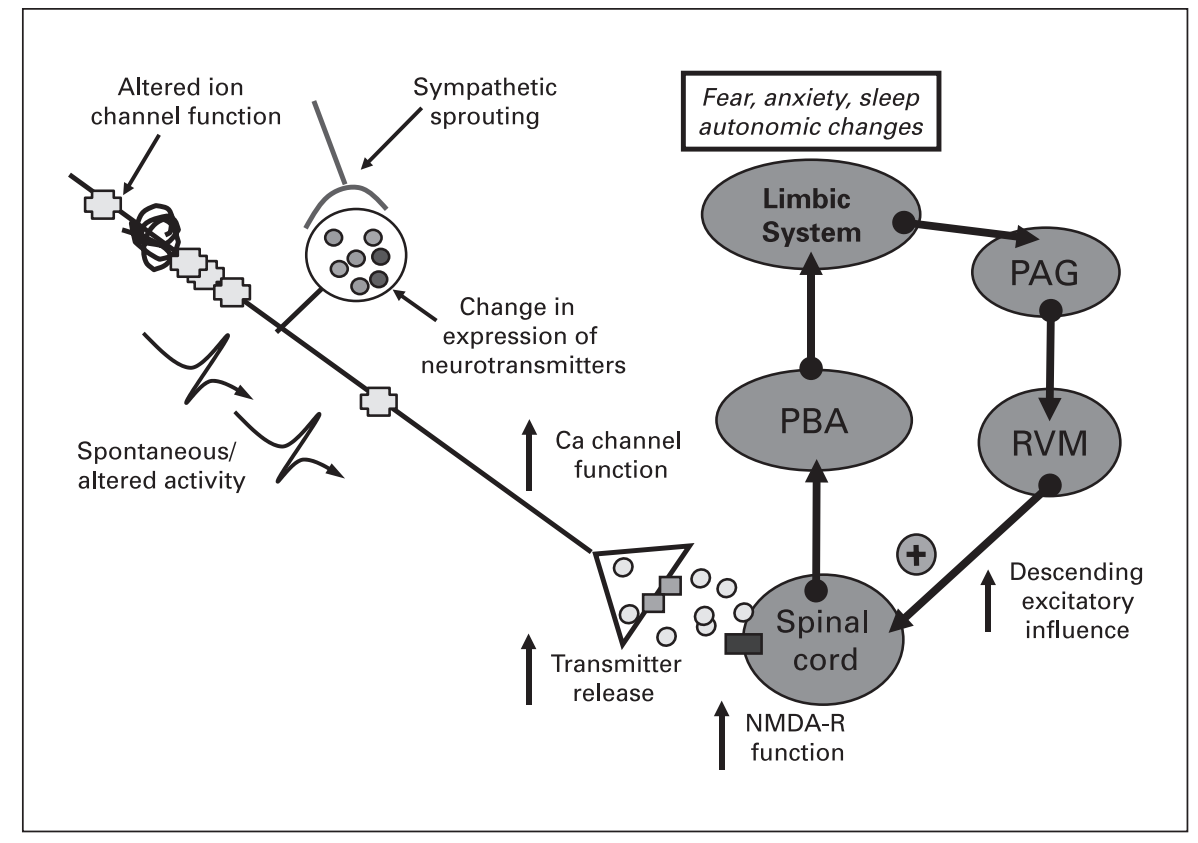

delta fibers are activated strongly by a 5HT3 agonist and a larger proportion of C-fibres (29\%) respond albeit with lower levels of firing [27]. In keeping with this characteristic, a recent study on sensory afferents from the colon showed that the majority were high-threshold afferents and 30\% responded to a selective 5HT3 agonist, 2-methyl-5HT [41]. 26\% of cell bodies retrogradely labelled from the colon displayed 5HT3 receptor-like immunoreactivity. The finding that the receptor is located on a subgroup of afferents suggests that the descending facilitations that act through this receptor would have selective effects. Moreover, the selectivity over mechanical vs. thermal inputs implicates a pre-synaptic mechanism since post-synaptic effects are likely to alter all activity. This pattern of effect agrees with what we have observed with SP-SAP and also fits well with behavioral data showing spinal section has a greater effect on mechanical allodynia than thermal responses in animals with nerve injury [42]. Furthermore, additional actions of 5HT may be mediated through post-synaptic and intrinsic systems in the dorsal horn $[25,26]$. Enkephalin-immunoreactive neurons in the superficial dorsal horn are innervated by 5-HT-immunoreactive fibers. Using single-cell RT-PCR 5HT3 receptor mRNA was detected in $\sim 25 \%$ of enkephalinergic neurons. Although some of the antinociception elicited by activation of 5HT3 receptors at the spinal cord may involve enkephalinergic dorsal horn neurons, this cannot explain the pronociceptive effects of this receptor.
The 5HT3 receptor has also been shown, by merit of its presynaptic location, to open calcium channels, causing long slow openings of these channels that would lead to increased transmitter release. Interestingly, presynaptic $5 \mathrm{HT} 3$ receptors are found throughout the brain, with differences in the kinetics of their responses in different brain regions [43]. Only a proportion of the synaptosomal population responded to 5HT3 agonists, suggesting that these receptors are able to regulate the functioning of select synapses. Afferent activity may also regulate the 5HT3 receptor since in vitro electrophysiological recordings revealed that calcium influx increases the cytoplasmic $\mathrm{Ca}^{2+}$ concentration and markedly reduces desensitization of the 5HT3 receptor channel [44]. Thus, there is cross-talk between voltage-gated $\mathrm{Ca}^{2+}$ channels and 5HT3 receptors. We therefore hypothesized that one consequence of activation of this serotonergic system would be an interaction with drugs that act to alter transmitter release. One candidate would be gabapentin (GBP), which is an anticonvulsant widely used for the treatment of various types of neuropathic pain [45-48]. The mechanism(s) of its anticonvulsant/analgesic actions remain to be determined. GBP binds to a unique site in the CNS identified as $\alpha_{2} \delta$ which is a modulatory accessory subunit of the voltage-dependent calcium channels (VDCC) [49, 50], and may regulate the function of VDCCs. Despite the fact that the $\alpha_{2} \delta$ subunit is common to all VDCCs, GBP is clinically efficacious against chronic pain with minimal 
adverse effects [51]. Behavioral studies show GBP is ineffective against physiological sensory nociception but produces robust antinociception following pathology such as nerve injury and inflammation [51-57]. In all of these studies GBP was effective against mechanical inputs; however, for thermal responses, mixed effects have been reported with the majority of positive effects being seen in inflammatory rather than neuropathic models.

We administered spinal ondansetron at an effective dose in the SNL induced neuropathic rats and observed the expected inhibition of evoked responses of the dorsal horn neurons. However the administration of a moderate dose of GBP, during the inhibition produced by block of the 5HT3-mediated facilitation, was now entirely without effect. Thus blockade of the 5HT3 receptor prevented GBP from inhibiting neuronal activity, suggesting that both agents may have similar pre-synaptic action. The depolarizing effects of 5HT3 receptor activation on afferent terminals may thus prolong calcium channel opening. This may then allow the interaction of GBP with the $\alpha_{2} \delta$ subunit to reduce VDCC activity and inhibit transmitter release. One functional commonality between the two drugs is their greater effectiveness on mechanical, rather than thermal activity, which fits with the lack of colocalization of the 5HT3 receptor and VR-1 on afferent fibers [27]. Furthermore, ablation of the NK-1 expressing neurones, ondansetron and GBP administration, and genetic deletion of 5HT3 receptor lead to very similar effects on the formalin response. It is unlikely that post-synaptic actions of any drug could underlie these selective effects on dorsal horn neuronal responses as the majority of both superficial and deep dorsal horn neurones respond to mechanical, thermal and chemical stimuli. However, one study did report differential activation of defined populations of dorsal horn neurons, suggestive of a separation of mechanical and thermal inputs. In lamina II of the dorsal horn, $15 \%$ of a population of neurons expressing the MOR 1 receptor responded (c-fos) to thermal stimulation but failed to respond to formalin or mechanical stimulation [58]. We propose that the interactions between the 5HT3 receptor and calcium channel modulation by GBP at presynaptic sites allow preferential actions on mechanical (and chemical)-evoked responses.

\section{Conclusions}

The anatomy and pharmacology of descending facilitation is not yet fully understood, nor the extent to which this mechanism underpins chronic pain states arising from peripheral neuropathy. Nonetheless, what is clear is that greater understanding and elucidation of pain pathways, their receptors and substrates, can pave the way towards improved pain therapy.

\section{Acknowledgements}

Our research is funded by the Wellcome Trust and the London Pain Consortium.

\section{References}

-1 Wang H, Sun H, Della Penna K, Benz R, Xu J, Gerhold D, Holder D, Koblan K: Chronic neuropathic pain is accompanied by global changes in gene expression and shares pathobiology with neurodegenerative diseases. Neuroscience 2002; 114:529-546.

2 Garry M, Tanelian D: Afferent activity in injured afferent nerves; in Yaksh T, Lynch $\mathrm{C}$, Zapol W, Maze M, Biebuyck J, Saidman L (eds): Anesthesia. Biologic Foundations. Philadelphia, Lippincott-Raven, 1997, pp 531542

- 3 Suzuki R, Dickenson A: Neuropathic pain: nerves bursting with excitement. Neuroreport 2000;11:R17-R21.

4 Sorkin L, Carlton S: Spinal anatomy and pharmacology of afferent processing; in Yaksh $\mathrm{T}$, Lynch C, Zapol W, Maze M, Biebuyck J, Saidman L (eds): Anesthesia. Biologic Foundations. Philadelphia, Lippincott-Raven, 1997, pp 577-610.
$>5$ Todd A: Anatomy of primary afferents and projection neurones in the rat spinal dorsal horn with particular emphasis on substance $P$ and the neurokinin 1 receptor. Expl Physiol 2002;87:245-249.

6 Woolf C, Fitzgerald M: The properties of neurones recorded in the superficial dorsal horn of the rat spinal cord. J Comp Neurol 1983;221: 313-328.

7 Craig A, Dostrovsky J: Differential projections of thermoreceptive and nociceptive lamina I trigeminothalamic and spinothalamic neurons in the cat. J Neurophysiol 2001;86:856-870.

-8 Marshall GE, Shehab SA, Spike RC, Todd AJ: Neurokinin-1 receptors on lumbar spinothalamic neurons in the rat. Neuroscience 1996; 72:255-263.

9 Light A, Sedivec M, Casale E, Jones S: Physiological and morphological characteristics of spinal neurones projecting to the parabrachial region of the cat. Somatosens Mot Res 1993; 10:309-325.
10 Hylden JL, Anton F, Nahin RL: Spinal lamina I projection neurons in the rat: collateral innervation of parabrachial area and thalamus. Neuroscience 1989;28:27-37.

11 Wall P, Bery J, Saade N: Effects of lesions to rat spinal cord lamina I cell projection pathways on reactions to acute and chronic noxious stimuli. Pain 1988;35:327-339.

12 Gauriau C, Bernard J: Pain pathways and parabrachial circuits in the rat. Exp Physiol 2002;87:251-258.

13 Bester H, Menendez L, Besson J, Bernard J: Spino (trigemino) parabrachiohypothalamic pathway: electrophysiological evidence for an involvement in pain processes. J Neurophysiol 1995; 73:568-585.

14 Hunt S, Mantyh P: The molecular dynamics of pain control. Nat Rev 2001;2:83-91.

15 Fields HL: Pain modulation: expectation, opioid analgesia and virtual pain. Prog Brain Res 2000;122:245-253. 
16 Millan MJ: Descending control of pain. Prog 31 Ikeda H, Heinke B, Ruscheweyh R, SandNeurobiol 2002;66:355-474.

17 Urban M, Gebhart G: Supraspinal contributions to hyperalgesia. Proc Natl Acad Sci USA 1999;95:2630-2635.

18 Zhuo M, Gebhart G: Biphasic modulation of spinal nociceptive transmission from the medullary raphe nuclei in the rat. J Neurophysiol 1997;78:746-758.

19 Urban MO, Gebhart GF: Characterisation of biphasic modulation of spinal nociceptive transmission by neurotensin in the rat rostral ventromedial medulla. J Neurophysiol 1997; 78:1550-1562.

20 Dickenson AH: A cure for wind up: NMDA receptor antagonists as potential analgesics. Trends Pharmacol Sci 1990;11:307-309.

-21 Herrero J, Laird J, Lopez-Garcia J: Wind-up of spinal cord neurones and pain sensation: much ado about something? Prog Neurobiol 2000;61:169-203.

-22 Mantyh P, Rogers S, Honore P, Allen B, Ghilardi J, Li J, Daughters R, Lappi D, Wiley $\mathrm{R}$, Simone D: Inhibition of hyperalgesia by ablation of lamina I spinal neurons expressing the substance P receptor. Science 1997;278:275279.

-23 Suzuki R, Morcuende S, Webber M, Hunt S, Dickenson A: Superficial NK1 expressing neurones control spinal excitability by activation of descending pathways. Nat Neurosci 2002;5: 1319-1326.

-24 Todd AJ, Spike RC, Polgar E: A quantitative study of neurons which express neurokinin-1 or somatostatin sst 2 a receptor in rat spinal dorsal horn. Neuroscience 1998;85:459-473.

-25 Morales M, Battenberg E, Bloom FE: Distribution of neurons expressing immunoreactivity for the 5HT3 receptor subtype in the rat brain and spinal cord. J Comp Neurol 1998;402: 385-401.

26 Maricq AV, Peterson AS, Brake AJ, Myers RM, Julius D: Primary structure and functional expression of the 5HT3 receptor, a serotonin-gated ion channel. Science 1991;254: $432-437$.

-27 Zeitz K, Guy N, Malmberg A, Dirajlal S, Martin W, Sun L, Bonhaus D, Stucky C, Julius D, Basbaum A: The 5-HT3 subtype of serotonin receptor contributes to nociceptive processing via a novel subset of myelinated and unmyelinated nociceptors. J Neurosci 2002;22:10101019.

$\checkmark 28$ Green G, Scarth J, Dickenson A: An excitatory role for 5-HT in spinal inflammatory nociceptive transmission: state-dependent actions via dorsal horn 5-HT3 receptors in the anaesthetized rat. Pain 2000;89:81-88.

-29 Ali Z, Wu G, Kozlov A, Barasi S: The role of 5-HT3 in antinociceptive processing in the rat spinal cord: results from behavioural and electrophysiological studies. Neurosci Lett 1996; 208:203-207.

- 30 Rygh L, Kontinen V, Suzuki R, Dickenson A: Different increase in C-fibre evoked responses after nociceptive conditioning stimulation in sham-operated and neuropathic rats. Neurosci Lett 2000;288:99-103. kuhler J: Synaptic plasticity in spinal lamina I projection neurons that mediate hyperalgesia. Science 2003;299:1237-1240.

-32 Willis W: Long-term potentiation in spinothalamic neurons. Brain Res Rev 2002;40:202214.

33 Ossipov M, Lai J, Malan TJ, Porreca F: Spinal and supraspinal mechanisms of neuropathic pain. Ann NY Acad Sci 2000;909:12-24.

34 Dickenson A, Matthews E, Suzuki R: Central nervous system mechanisms of pain in peripheral neuropathy; in Hansson P, Fields H, Hill R, Marchettini P (eds): Neuropathic Pain: Pathophysiology and Treatment. Seattle, IASP Press, 2001, pp 85-106.

-35 Zimmermann M: Pathobiology of neuropathic pain. Eur J Pharmacol 2001;429:23-37.

36 Burgess S, Gardell L, Ossipov M, Malan T Jr, Vanderah T, Lai J, Porreca F: Time-dependent descending facilitation from the rostral ventromedial medulla maintains, but does not initiate, neuropathic pain. J Neurosci 2002;22: 5129-5136.

37 Porreca F, Ossipov M, Gebhart G: Chronic pain and medullary descending facilitation. Trends Neurosci 2002;25:319-325.

38 Heinricher M, Roychowdhury S: Reflex-related activation of putative pain facilitating neurones in rostral ventromedial medulla requires excitatory amino acid transmission. Neuroscience 1997;78:1159-1165.

39 Monassi CR, Bandler R, Keay KA: A subpopulation of rats show social and sleep-waking changes typical of chronic neuropathic pain following peripheral nerve injury. Eur J Neurosci 2003; 17:1907-1920.

40 Farber L, Stratz T, Bruckle W, Spath M, Pongratz D, Lautenschlager J, Kotter I, Zoller B, Peter H, Neeck G, Alten R, Muller W: Efficacy and tolerability of tropisetron in primary fibromyalgia: a highly selective and competitive 5HT3 receptor antagonist. Scand J Rheumatol Suppl 2000;113:49-54.

41 Hicks GA, Coldwell JR, Schindler M, Ward PA, Jenkins D, Lynn PA, Humphrey PP, Blackshaw LA: Excitation of rat colonic afferent fibres by 5-HT(3) receptors. J Physiol 2002; 544:861-869.

42 Bian D, Ossipov MH, Zhong C, Malan TP, Jr., Porreca F: Tactile allodynia, but not thermal hyperalgesia, of the hindlimbs is blocked by spinal transection in rats with nerve injury. Neurosci Lett 1998;241:79-82.

-43 Nayak SV, Ronde P, Spier AD, Lummis SC, Nichols RA: Calcium changes induced by presynaptic 5-hydroxytryptamine-3 serotonin receptors on isolated terminals from various regions of the rat brain. Neuroscience 1999;91: 107-117.

44 Jones S, Yakel J: $\mathrm{Ca}^{2+}$ influx through voltagegated $\mathrm{Ca}^{2+}$ channels regulates 5-HT3 receptor channel desensitization in rat glioma $\mathrm{x}$ mouse neuroblastoma hybrid NG108-15 cells. J Physiol 1998;510:361-370.

45 Nicholson B: Gabapentin use in neuropathic pain syndromes. Acta Neurol Scand 2000;101: 359-371.
46 Serpell MG: Gabapentin in neuropathic pain syndromes: a randomised, double-blind, placebo-controlled trial. Pain 2002;99:557-566.

47 Backonja MM: Use of anticonvulsants for treatment of neuropathic pain. Neurology 2002;59:S14-S17.

48 Rose MA, Kam PC: Gabapentin: pharmacology and its use in pain management. Anaesthesia 2002; 57:451-462.

49 Gee NS, Brown JP, Dissanayake VU, Offord J, Thurlow R, Woodruff GN: The novel anticonvulsant drug, gabapentin (Neurontin), binds to the alpha2delta subunit of a calcium channel. J Biol Chem 1996;271:5768-5776.

50 Field MJ, Hughes J, Singh L: Further evidence for the role of the alpha(2)delta subunit of voltage dependent calcium channels in models of neuropathic pain. Br J Pharmacol 2000;131: 282-286.

51 Field MJ, Oles RJ, Lewis AS, McCleary S, Hughes J, Singh L: Gabapentin (neurontin) and S-(+)-3-isobutylgaba represent a novel class of selective antihyperalgesic agents. $\mathrm{Br} \mathrm{J}$ Pharmacol 1997;121:1513-1522.

52 Abdi S, Lee DH, Chung JM: The anti-allodynic effects of amitriptyline, gabapentin, and lidocaine in a rat model of neuropathic pain. Anesth Analg 1998;87:1360-1366.

53 Hunter JC, Gogas KR, Hedley LR, Jacobson LO, Kassotakis L, Thompson J, Fontana DJ: The effect of novel anti epileptic drugs in rat experimental models of acute and chronic pain. Eur J Pharmacol 1997;324:153-160.

54 Erichsen HK, Blackburn-Munro G: Pharmacological characterisation of the spared nerve injury model of neuropathic pain. Pain 2002; 98:151-161.

55 Hwang JH, Yaksh TL: Effect of subarachnoid gabapentin on tactile-evoked allodynia in a surgically induced neuropathic pain model in the rat. Reg Anesth 1997;22:249-256.

56 Yoon MH, Yaksh TL: The effect of intrathecal gabapentin on pain behavior and hemodynamics on the formalin test in the rat. Anesth Analg 1999;89:434-439.

57 Christensen D, Gautron M, Guilbaud G, Kayser V: Effect of gabapentin and lamotrigine on mechanical allodynia-like behaviour in a rat model of trigeminal neuropathic pain. Pain 2001;93:147-153.

58 Spike RC, Puskar Z, Sakamoto H, Stewart W, Watt C, Todd AJ: MOR-1-immunoreactive neurons in the dorsal horn of the rat spinal cord: evidence for nonsynaptic innervation by substance P-containing primary afferents and for selective activation by noxious thermal stimuli. Eur J Neurosci 2002;15:1306-1316.

59 Mccleane G, Suzuki R, Dickenson A: Does a single intravenous injection of the $5 \mathrm{HT} 3$ receptor antagonist ondansetron have an analgesic effect in neuropathic pain? A double-blinded, placebo-controlled cross-over study. Anesth Analg 2003;97:1474-1478.

-60 Suzuki R, Rahman W, Hunt S, Dickenson A: Descending facilitatory control of mechanically evoked responses is enhanced in deep dorsal horn neurones following peripheral nerve injury. Brain Res 2004;1019:68-76. 